\title{
MODELOS BIOLÓGICOS DE CRESCIMENTO EM DIÂMETRO PARA AROEIRA
}

\author{
Carlos Roberto Sanquetta ${ }^{1}$; Aline Durigan²; Mateus Niroh Inoue Sanquetta ${ }^{2}$; Ana \\ Paula Dalla Corte ${ }^{1}$; Greyce Charllyne Benedet Maas ${ }^{3}$ \\ 1 - Engenheiro Florestal, Doutor, Docente da Universidade Federal do Paraná (UFPR) \\ (carlossanquetta@gmail.com) \\ 2 - Discente de Engenharia Florestal da UFPR \\ 3 - Tecnólogo Ambiental, Dr., Bolsista do CNPq na UFPR Avenida Lothário Meissner, 900 - \\ Jardim Botânico Curitiba - PR, 80.210-170 - Brasil
}

Recebido em: 08/04/2016 - Aprovado em: 30/05/2016 - Publicado em: 20/06/2016 DOI: 10.18677/Enciclopedia_Biosfera_2016_070

\begin{abstract}
RESUMO
Analisou-se o crescimento em diâmetro de 30 indivíduos representativos de aroeira em um povoamento experimental homogêneo com 11 anos de idade instalado em Pinhais - PR. O método empregado para análise do crescimento foi a ANATRO (análise de tronco) de discos do fuste tomados à altura de 1,30 m, nos quais foram contados e medidos os anéis anuais de crescimento com uso de lupa. Modelos biológicos de crescimento foram testados e as respectivas equações ajustadas. Os ajustes não lineares foram realizados utilizando-se o Solver $^{\circledR}$ do MSExce ${ }^{\circledR}$. O incremento médio anual em diâmetro variou de 6,53 a $9,79 \mathrm{~mm}$, respectivamente nas idades 1 e 10, ou seja, o maior incremento ocorreu na fase inicial (primeiro ano) e o menor no último ano, com comportamento decrescente curvilíneo. $O$ comportamento do crescimento se mostrou classicamente sigmoide, porém sem indicativo do atingimento da assíntota até a idade de 10 anos. Os ajustes com os modelos biológicos de crescimento resultaram em estimativas acuradas, porém com baixa precisão devido à dispersão da nuvem de pontos. O modelo de ChapmanRichards foi indicado como o de melhor performance. Concluiu-se que houve formação clara de anéis anuais de crescimento na espécie, que o crescimento em diâmetro ainda não atingiu seu máximo, que há grande dispersão de crescimento entre indivíduos e que os modelos biológicos descrevem apropriadamente (sem viés) o comportamento da curva de crescimento em diâmetro da espécie estudada.
\end{abstract}

PALAVRAS-CHAVE: análise de tronco, aroeira-pimenteira, incremento.

\section{BIOLOGICAL MODELS FOR DIAMETER GROWTH OF THE BRAZILIAN PEPPER-TREE}

\begin{abstract}
We analyzed the diameter growth of 30 representative individuals of the Brazilian pepper-tree in a homogeneous experimental stand of 11 years of age established in Pinhais - PR. The method used to analyze the growth was stem analysis through discs of the boles taken at $1.3 \mathrm{~m}$ from the ground level, in which annual growth rings were counted and measured the with use of a magnifier. Biological growth models were tested and respective equations were obtained. Nonlinear fitting was performed by using the MSExcel ${ }^{\circledR}$ Solver ${ }^{\circledR}$. The average annual diameter increment ranged from 6.53 to $9.79 \mathrm{~mm}$, respectively at ages 1 and 10, i.e., the largest increment happened
\end{abstract}


in the initial phase (first year) and the lowest in the last year, showing a decreasing curvilinear behavior. The growth behavior proved classically sigmoid, but with no indication of reaching the asymptote to the age of 10 years. Biological models provided accurate estimates, but with low precision due to high dispersion of growth. The Chapman-Richards model showed the best performance. It was concluded the species form clearly annual growth rings, the diameter growth has not yet reached its maximum rate, there is great variation in growth rate among individuals, and biological models properly (without bias) describe the behavior of the growth curve diameter of the species studied.

KEYWORDS: stem analysis, pepper-tree, increment.

\section{INTRODUÇÃO}

Genericamente o termo crescimento designa o aumento das dimensões. Especificamente para árvores e povoamentos florestais, trata-se de um processo de mudança na forma e tamanho da árvore, expressando quantidades das variáveis biométricas num determinado tempo (CAMPOS \& LEITE, 2009).

Existem vários métodos para analisar o crescimento de árvores e povoamentos florestais, cada um com suas vantagens e desvantagens, sendo muitas vezes empregados complementarmente. A análise de tronco (ANATRO) é uma técnica muito útil quando as árvores formam anéis de crescimento anuais claros. A técnica vem sendo usada há muito tempo, desde o fim do século XIX e início do século $X X$, para elaboração de tabelas de produção florestal com fins comerciais (MACHADO et al., 2013) e com vários outros objetivos.

Outra técnica muito utilizada na atualidade para expressar o crescimento florestal são os modelos de crescimento e produção. Tais modelos são abstrações de um sistema real, com aplicações variadas, seja em inventários florestais, construção de tabelas de produção, indicação de regimes de manejo, etc. (TITUS \& MARTON, 1988). Independentemente da categoria, os modelos de crescimento e produção baseiam-se em ajustes realizados com a aplicação da técnica de regressão linear ou não linear (CASTRO et al., 2013).

Modelos são amplamente utilizados na área florestal, notadamente para descrever e prognosticar o crescimento. Historicamente, modelos empíricos foram bastante utilizados, mas mais recentemente ênfase vem sendo dada aos modelos biológicos, devido ao seu potencial explicativo dos processos biológicos por detrás das expressões matemáticas, ou seja, seu realismo biológico. Vários modelos biológicos vêm sendo utilizados na ciência florestal ao longo dos anos, entre os quais os de Chapman-Richards, Gompertz, Logística e Monomolecular. Turnbull, em 1963, foi o primeiro a aplicar os modelos biológicos na biometria florestal (ZANIN, 2013).

Apesar de muitos estudos sobre crescimento e produção florestal já terem sido realizados no Brasil e no mundo, atualmente, constata-se uma carência de informações sobre o crescimento de espécies nativas, sobretudo pela carência de observações de longa duração (SPATHELF et al., 2000).

Schinus terebinthifolius Raddi (aroeira ou aroeira-pimenteira), uma das espécies mais cosmopolitas da flora regional do sul do Brasil, apresenta ocorrência em toda a costa brasileira, particularmente no bioma Mata Atlântica (MARTORELLI et al., 2011). Tem rápido crescimento e potencial econômico (madeireiro e não madeireiro) e ambiental (CORADIN et al., 2011; SANQUETTA et al., 2015a). Contudo, plantios experimentais com a espécie e dados sobre seu crescimento em condições de povoamento são praticamente inexistentes. 
Nesta pesquisa é analisado o crescimento em diâmetro de árvores individuais da espécie em um experimento, utilizando-se as técnicas de ANATRO e modelos biológicos de crescimento, almejando dar elementos para o futuro manejo e utilização da espécie em maior escala.

\section{MATERIAL E MÉTODOS}

A área de estudo é a Fazenda Experimental do Canguiri, situada em Pinhais $\mathrm{PR}$, que pertence à Universidade Federal do Paraná, sob as coordenadas $25^{\circ} 23^{\prime}$ 30 " S de latitude e $49^{\circ} 07^{\prime} 30^{\prime \prime} \mathrm{W}$ de longitude. A a ltitude local é de $900 \mathrm{~m}$ e o tipo climático é $\mathrm{Cfb}$, conforme Koeppen. Maiores detalhes podem sobre área ser obtidos em RIBEIRO et al. (2008) e SANQUETTA et al. (2015b).

A espécie estudada é a aroeira (Schinus terebinthifolius Raddi), uma árvore pertencente à família Anacardiaceae da flora nativa do Brasil. Ocorre naturalmente na América do Sul, ao longo de toda a costa brasileira, particularmente na Mata Atlântica (MARTORELLI et al., 2011). Ela ocorre amplamente e possui rápido crescimento, preferindo áreas sem vegetação e, apresentando em alguns casos, caráter invasor (D’ÁVILA et al., 2010). Possui usos madeireiros e não madeireiros, sendo ainda recomendada para restaurar de áreas alteradas (FERRETI et al., 1995).

Foram selecionados 30 indivíduos representativos do experimento implantado em 2003, ou seja, com 11 anos de idade à época do levantamento (dezembro de 2014). Maiores detalhes da área experimental são encontrados em SANQUETTA et al. (2015a,b).

A seleção dos exemplares foi feita com base na distribuição de diâmetros do povoamento. As plantas foram cortadas e discos do fuste foram retirados a $1,30 \mathrm{~m}$ do solo (dap). Os discos foram secos em estufa e lixados para melhor visualização dos anéis de crescimento. Foi marcado o maior raio do disco e tirado um ângulo de 45 para traçar quatro raios na peça. Os anéis de crescimento foram contados e medidos com lupa com régua graduada interna.

Os raios identificados e registrados nos discos de madeira foram convertidos em diâmetros sem casca para todas as idades, multiplicando-se por dois. Utilizandose a relação entre diâmetro com casca e sem casca dos discos foram estimados todos os diâmetros com casca em toda a amplitude de idades das 30 árvores amostradas. A equação da reta foi usada, tendo em vista a tendência retilínea da nuvem de pontos.

As curvas de crescimento de todas as 30 árvores foram então obtidas a partir dos incrementos anuais, gerando uma nuvem de pontos do diâmetro à altura do peito com casca em função da idade em anos. A média e o coeficiente de variação dos incrementos médios anuais foram calculados a partir dos dados.

Para descrever o crescimento passado e possibilitar prognoses futuras, foram testados cinco modelos biológicos reconhecidos na literatura correlata (quadro 1).

QUADRO 1 - Modelos biológicos testados para dados de dap e idade em aroeira em Pinhais - PR

\begin{tabular}{lc}
\hline Modelo & Formulação \\
\hline Bertalanffy & $d=a(1-\exp (-b . I))^{2 / 3}$ \\
Chapman-Richards & $d=a(1-\exp (-b . I))^{c}$ \\
Gompertz & $d=a \exp (-b \exp (-c . l))$ \\
Korf & $\left.d=a \exp \left(-b . I^{-c}\right)\right)$ \\
Logística & $d=a /(1-b \exp (-c . I))$ \\
\hline
\end{tabular}


Sendo:

$\mathrm{d}=$ diâmetro a 1,30 do solo - dap (mm);

$\mathrm{I}=$ idade (anos);

$a, b, c=$ coeficientes a estimar por regressão não linear.

O desempenho dos ajustes dos modelos retrocitados foi avaliado segundo três indicadores numéricos relativos à média (PRETZSCH, 2009; BURKHART \& TOMÉ, 2012) e pelos gráficos de ajuste. Os critérios numéricos foram os seguintes:

Viés:

$\bar{e} \%=\frac{\bar{e}}{\bar{x}} 100$

sendo: $\bar{e}=\frac{\sum_{i=1}^{n}\left(\hat{x}_{i}-x_{i}\right)}{n}$

Precisão:

$s_{e} \%=\frac{s_{e}}{\bar{x}} 100(3)$

Sendo: $s_{e}=\sqrt{\frac{\sum_{i=1}^{n}\left(\hat{x}_{i}-\bar{e}-x_{i}\right)^{2}}{n-1}}(4)$

e

Acurácia:

$m_{x} \%=\frac{m_{x}}{\bar{x}} 100(5)$

sendo: $m_{x}=\sqrt{\frac{\sum_{i=1}^{n}\left(\hat{x}_{i}-x_{i}\right)^{2}}{n-1}}(6)$

em que:

$\hat{x}_{i}=$ valor estimado do dap $(\mathrm{mm})$;

$x_{i}=$ valor real do dap $(\mathrm{mm})$;

$\bar{x}=$ valor médio real do dap $(\mathrm{mm})$;

$n=$ número de dados.

Quanto mais próximo de zero (em termos modulares) menor é o viés $\bar{e} \%$ e melhor é o desempenho do modelo. Quanto menor a dispersão expressa por $s_{e} \%$ melhor é o modelo e maior a precisão. Quanto menor $m_{x} \%$ maior a acurácia e mais próximo do alvo estão as estimativas. A interpretação desses indicadores pode ser visualizada na Figura 1, fazendo-se uma analogia com tiros em um alvo. 


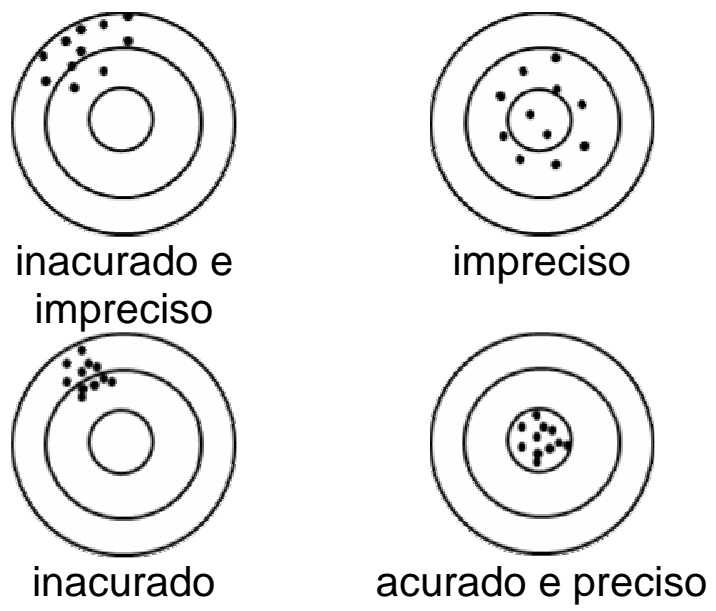

FIGURA 1 - Representação da performance de modelos segundo a precisão e acurácia em analogia a um alvo

\section{RESULTADOS E DISCUSSÕES}

\section{Estatísticas dos Incrementos}

Os anéis de crescimento dos discos de madeira retirados mostraram-se bem visíveis e anuais, fato comprovado pelo registro do plantio. Assim foi possível marcar e medir corretamente os anéis e avaliar o crescimento das árvores amostradas neste estudo.

Em média, o dap atingido pelas árvores, após 10 anos de crescimento, foi de $65,30 \mathrm{~mm}$, com IMA de $6,53 \mathrm{~mm}$.ano ${ }^{-1}$. Verificou-se variação considerável nos diâmetros das árvores, expressa pelo coeficiente de variação (CV\%), sobretudo na fase inicial de crescimento (Tabela 2). A causa disso, possivelmente, seja o material genético das mudas. Procurou-se utilizar mudas padronizadas e das mesmas matrizes, mas não foi possível maior controle do material genético no experimento. Não se atribuem essas diferenças de crescimento às variações do sítio, pois o local do experimento é pequeno é aparentemente bastante homogêneo quanto ao relevo, solo e microclima.

TABELA 2-Estatísticas do crescimento em dap e idade em aroeira em Pinhais - PR

\begin{tabular}{ccccccccccc}
\hline Idade & 1 & 2 & 3 & 4 & 5 & 6 & 7 & 8 & 9 & 10 \\
\hline Média & 9,79 & 16,75 & 24,03 & 30,19 & 36,81 & 43,16 & 49,89 & 55,36 & 58,28 & 65,30 \\
CV\% & 40,88 & 26,83 & 28,01 & 25,19 & 22,25 & 21,04 & 24,52 & 12,50 & 8,16 & 11,57 \\
Mínimo & 3,69 & 9,25 & 12,83 & 18,00 & 21,97 & 26,34 & 28,33 & 45,82 & 51,78 & 56,95 \\
Máximo & 18,79 & 30,72 & 39,46 & 49,80 & 57,75 & 65,70 & 82,39 & 65,30 & 63,18 & 71,66 \\
IMA & 9,79 & 8,37 & 8,01 & 7,55 & 7,36 & 7,19 & 7,13 & 6,92 & 6,48 & 6,53 \\
\hline Nota: idade & anos, & valores da média & mínimo e máximo & mm & IMA
\end{tabular}
(incremento médio annual em mm.ano ${ }^{-1}$ )

As curvas de crescimento individual em dap mostraram comportamento sigmoide até a idade considerada, porém não se observa tendência de atingimento da assíntota até 10 anos de idade. A maioria das árvores não teve a curva completa até 10 anos devido ao fato de que os discos foram tomados a $1,30 \mathrm{~m}$ e não na base da árvore. Disso decorre que em alguns casos foram necessários até 3 anos para que as mudas atingissem 1,30 $\mathrm{m}$ de altura (Figura 2). 


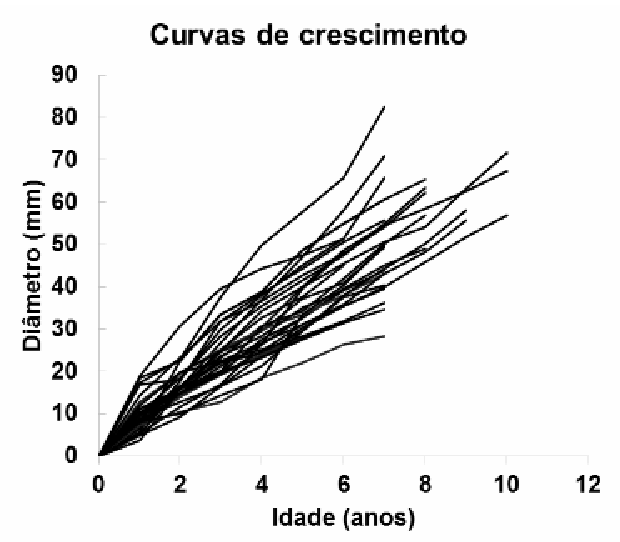

FIGURA 2 - Curvas de crescimento em dap e idade em aroeira em Pinhais - PR

\section{Modelos Biológicos}

Os modelos analisados mostraram baixos valores de viés quando do ajuste. Os menores valores de viés foram obtidos no ajuste dos modelos de ChapmanRichards e Korf, com superioridade do primeiro. Os outros modelos apresentaram leve tendência superestimativa, ou seja, viés positivo (Tabela 3). Os valores indicativos de acurácia foram apenas razoáveis, tendo em vista que seria desejável que fossem mais próximos de zero ou no máximo até $10 \%$, como regra. Acurácia da magnitude de $20-30 \%$ como verificado neste estudo também é reflexo da alta variabilidade das taxas de crescimento, como observado na Figura 3. Isso também tem reflexo na grande dispersão na nuvem de pontos dos valores de dap em função da idade, o que significa que a precisão das estimativas não foi elevada. Possivelmente será necessário estratificar os dados em futuras análises, mas esse não foi o foco deste estudo. Ademais, o número de árvores amostradas limita essa estratificação.

TABELA 3 - Viés, precisão e acurácia de modelos biológicos testados para dados de dap e idade de aroeira em Pinhais - PR

\begin{tabular}{lrccccc}
\hline Modelo & \multicolumn{1}{c}{$\mathrm{a}$} & $\mathrm{b}$ & $\mathrm{c}$ & $\bar{e} \%$ & $s_{e} \%$ & $m_{x} \%$ \\
\hline Bertalanffy & $2.629,3897$ & 0,0003 & - & 2,74 & 197,39 & 26,66 \\
Chapman-Richards & 968,5470 & 0,0044 & 0,8529 & $-0,02$ & 177,88 & 25,17 \\
Gompertz & 79,0716 & 2,5560 & 0,2436 & 2,69 & 192,41 & 26,32 \\
Korf & $29.094,4955$ & 8,1297 & 0,1240 & $-0,21$ & 178,59 & 25,23 \\
Logística & 66,9196 & $-7,4056$ & 0,4428 & 3,60 & 204,12 & 27,21 \\
\hline
\end{tabular}



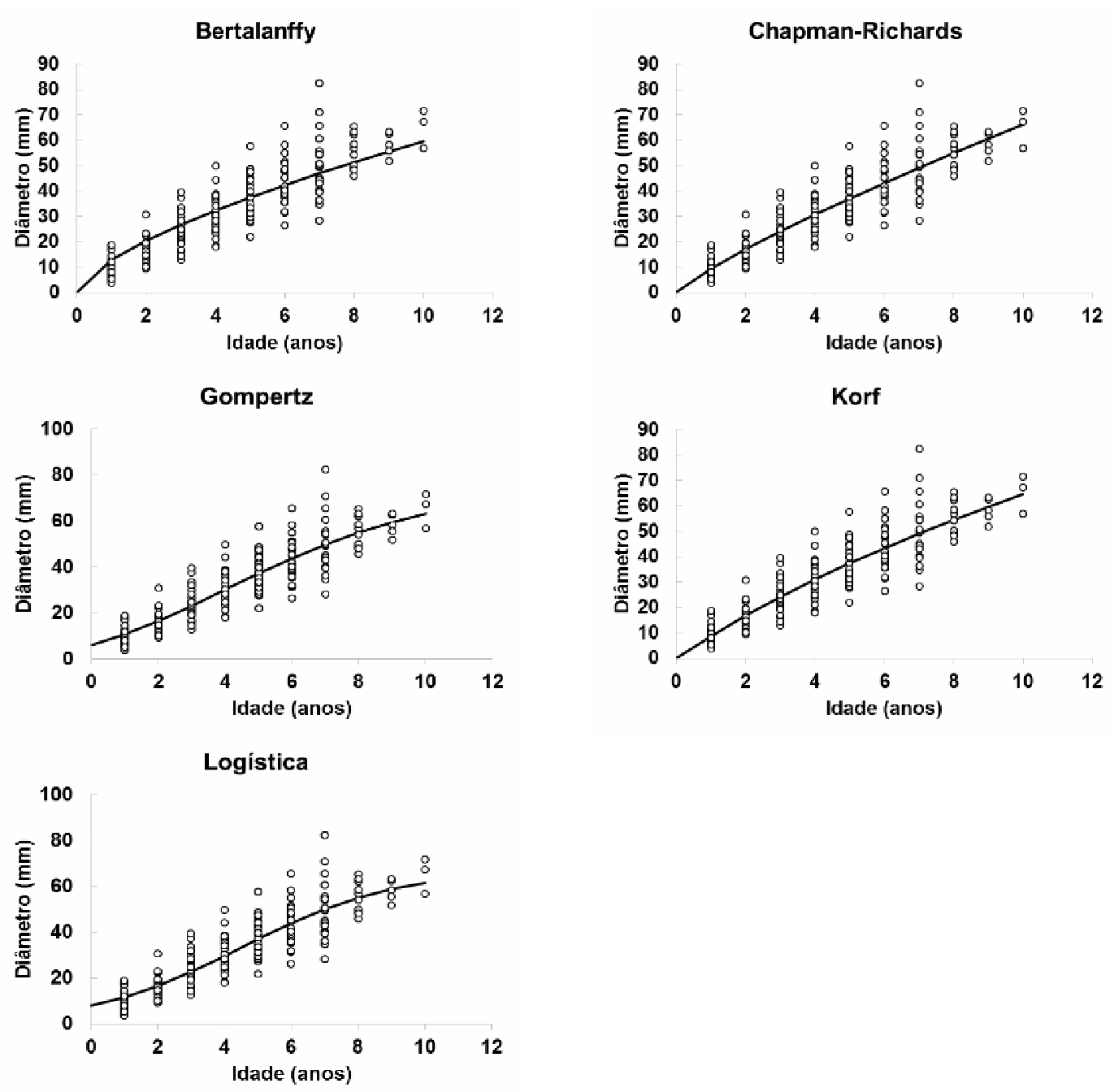

FIGURA 3 - Modelos biológicos testados para dados de dap e idade em aroeira em Pinhais - PR

Nota: círculos indicam valores reais e linha contínua indica a estimativa a partir da equação ajustada

\section{DISCUSSÕES}

A maioria das plantações florestais no Brasil é de espécies exóticas, dos gêneros Eucalyptus e Pinus (IBÁ, 2015). São poucas as espécies nativas plantadas para fins econômicos. Isso se deve principalmente à falta de pesquisa sobre as espécies da flora nativa brasileira e de um pacote tecnológico capaz de estimular investimentos em plantios comerciais dessas espécies. Uma das maiores restrições nesse contexto diz respeito à falta de informações sobre o crescimento das espécies com potencial econômico em condições experimentais.

Existem alguns estudos experimentais sobre o comportamento de algumas espécies florestais nativas, demonstrando o seu potencial crescimento (e.g. CARVALHO, 1994; WISCH \& MELLO, 2005; SOUZA, 2012). A maioria das pesquisas científicas sobre espécies florestais nativas, encontradas na literatura, é atinente à fase de germinação das sementes e produção de mudas, havendo apenas pequena parcela destinada a avaliar 0 potencial silvicultural em povoamentos puros (SOUZA, 2012).

Não existem na literatura experimentos sobre o comportamento do crescimento da aroeira em condições de plantio puro em campo. O crescimento da 
espécie foi analisado em condições naturais em Sergipe (MATTOS et al., 2012), que demonstraram que o IMA (incremento médio anual) em diâmetro atinge 0,60 cm.ano ${ }^{1}$ e ICA (incremento corrente anual) máximo de $0,96 \mathrm{~cm}$.ano ${ }^{-1}$ aos 14 anos. Mediante o ajuste do modelo biológico de Gompertz, os autores concluíram que o IPA (incremento periódico anual) nos últimos 5 anos, considerando indivíduos com idade máxima de 40 anos, era de $0,21 \mathrm{~cm}^{-a n o^{-1}}$. Os autores obtiveram bom ajuste desse modelo, mas não testaram outros para comparar.

Modelos biológicos de crescimento possuem algumas propriedades que os distinguem. A principal diferença entre um modelo empírico e um biológico é que o primeiro é apenas uma expressão matemática da relação entre variáveis, já um modelo biológico traz uma hipótese sobre a biologia do processo de crescimento (ZANIN, 2013). Ademais, os coeficientes dos modelos biológicos têm propriedades e significados biológicos. Por isso esses modelos vêm sendo utilizados não só na ciência florestal, como por exemplo, para retratar o crescimento de vacas leiteiras (SILVA et al., 2011), em crescimento do tomateiro (MENDES, 2011), entre outros.

$\mathrm{Na}$ área florestal os modelos biológicos vêm sendo muito utilizados com sucesso, desde o trabalho pioneiro de PIENAAR (1965), que analisou o significado biológico dos coeficientes e a relação entre eles nos distintos modelos teóricos. Particularmente no presente caso, os modelos podem ser empregados na prática para descrever o comportamento da curva de crescimento em diâmetro da espécie alvo e fazer projeções de curto a médio prazo, visando apontar qual será o provável crescimento nos próximos anos. Isso é útil para indicar a produtividade florestal em plantios comerciais com a espécie.

\section{CONCLUSÕES}

- Há formação clara de anéis anuais de crescimento na espécie, segundo a experiência da leitura e medição dos discos tomados neste estudo;

- Até a idade 10 anos ainda não houve o atingimento da assíntota no valor do dap, o que indica que as árvores continuam em ritmo acelerado de crescimento;

- Há grande dispersão de crescimento entre indivíduos da espécie em condições experimentais, a despeito da homogeneidade no sítio;

- Os modelos biológicos descrevem acuradamente (sem viés) 0 comportamento da curva de crescimento em diâmetro da espécie estudada, mas a alta dispersão das taxas de crescimento implica apenas em uma mediana precisão;

- Recomenda-se aprofundar a modelagem, utilizando alguns mecanismos de estratificação dos dados.

\section{REFERÊNCIAS}

BURKHART, H. E.; TOMÉ, M. Modeling forest trees and stands. New York: Springer, 2012. 460p.

CAMPOS, J. C. C.; LEITE, H. G. Mensuração florestal: perguntas e respostas. 3. Ed. Viçosa: Editora UFV, 2009. 548 p.

CARVALHO, P.E.R. Espécies florestais brasileiras; recomendações silviculturais, potencialidades e uso da madeira. Colombo: EMBRAPACNPFlorestas; Brasília: EMBRAPA - SPI, 1994. 640p. 
CASTRO, R. V. O.; SOARES, C. P. B.; MARTINS, F. B.; LEITE, H. G. Crescimento e produção de plantios comerciais de eucalipto estimados por duas categorias de modelos. Pesquisa Agropecuária Brasileira, v.48, n.3, p.287-295, 2013.

CORADIN, L.; SIMINSKI, A.; REIS, A. Espécies nativas da flora brasileira de valor econômico atual ou potencial: plantas para o futuro - Região Sul, Brasília: MMA, 934p. 2011.

D'AVILA, G.; GOMES JR., A.; CANARY, A. C.; BUGONI, L. The role of avian frugivores on germination and potential seed dispersal of the Brazilian Pepper Schinus terebinthifolius. Biota Neotropica, v. 10, n. 3, p.45-51, 2010. Disponível em: <http://dx.doi.org/10.1590/S1676-06032010000300004>. doi: 10.1590/S167606032010000300004.

FERRETTI, A. R.; KAGEYAMA, P. Y.; ÁRBOEZ, G. F.; SANTOS, J. D.; BARROS, M. I. A.; LORZA, R. F.; OLIVEIRA, C. Classificação das espécies arbóreas em grupos ecofisiológicos para revegetação com nativas no estado de São Paulo. Florestar Estatístico, v. 3, n. 7, p. 73-77, 1995.

IBÁ - INDÚSTRIA BRASILEIRA DE ÁRVORES. Indicadores de desempenho do setor nacional de árvores plantadas referentes ao ano de 2014. Brasília, 2015. $64 p$.

MACHADO, S. A.; SILVA, L. C. R.; JASKIU, E.; CAVALHEIRO, R. Comparação entre análise de tronco digital e convencional em árvores de Mimosa scabrella Bentham e Pinus taeda L. Revista Árvore, v.37, n.2, p.329-337, 2013. Disponível em: <http://dx.doi.org/10.1590/S0100-67622013000200014>. doi: 10.1590/S010067622013000200014.

MARTORELLI, S. B. F.; PINHEIRO, A.L.B.; HIGINO, J.S.; BRAVO, F. Efeito antiinflamatório e cicatrizante de extrato de hidroalcoólico de Schinus terebinthifolius Raddi (aroeira) a 30\% em ora base - estudo "in vivo". International Journal of Dentistry, v. 10, n. 2, p.80-90, 2011.

MATTOS, P. P.; DOMENE, V. D.; SANTOS, A. T.; MELLO, A. A.; SALIS, S. M.; FERREIRA, R. A.; SILVA, W. P. Estimativa de crescimento em diâmetro e volume. In: CONGRESSO FLORESTAL PARANAENSE, 4, 2012, Curitiba. Anais... 161-177. 2012.

MENDES, P. N. Ajuste dos modelos Gompertz e Logístico na descrição das curvas de crescimento de tomates: uma abordagem bayesiana. $82 \mathrm{f}$. Tese (Doutorado em Estatística e Experimentação Agropecuária), Universidade Federal de Lavras, Lavras, 2011.

PIENAAR, L. V. Quantitative theory of forest growth. Tese (Doutorado). University of Washington, 1965.167p.

PRETZSCH, H. Forest dynamics, growth and yield. München: Springer, 2009. $664 p$. 
RIBEIRO, L.; KOPROSKI, L. P.; STOLLE, L.; LINGNAU, C.; SOARES, R. V.; BATISTA, A. C. Zoneamento de riscos de incêndios florestais para a Fazenda Experimental do Canguiri, Pinhais (PR). Floresta, v.38, n.3, p. 561- 572, 2008. Disponível em: <http://dx.doi.org/10.5380/rf.v38i3.12430>. doi: 10.5380/rf.v38i3.12430.

SANQUETTA, M. N. I.; SANQUETTA, C.R.; SANTOS, A. B.; COUTINHO, V. M.; DALLA CORTE, A. P. Densidade da madeira de Schinus terebinthifolius Raddi. em povoamento puro no município de Pinhais - PR. Enciclopédia Biosfera, v. 11, n. 22, p. 3604-3612, 2015a. Disponível em: $<$ http://dx.doi.org/10.18677/Enciclopedia Biosfera 2015 122>. doi: 10.18677/Enciclopedia_Biosfera_2015_122.

SANQUETTA, M. N. I.; SANQUETTA, C.R.; DURIGAN, A.; DALLA CORTE, A. P.; COUTINHO, V. M.; Equações de biomassa para aroeira-pimenteira (Schinus terebinthifolius Raddi). Enciclopédia Biosfera, v. 11, n. 22, p. 832-840, 2015b. Disponível em: <http://dx.doi.org/10.18677/Enciclopedia Biosfera 2015 248>. doi: 10.18677/Enciclopedia_Biosfera_2015_248.

SILVA, F. L.; ALENCAR, M. M. FREITAS, A. R.; PACKER, I. U.; MOURÃO, G. B. Curvas de crescimento em vacas de corte de diferentes tipos biológicos. Pesquisa Agropecuária Brasileira, v.46, n.3, p.262-271, 2011. Disponível em: <http://dx.doi.org/10.1590/S0100-204X2011000300006>. doi: 10.1590/S0100204X2011000300006.

SOUZA, M. G. O. S. Crescimento de espécies florestais em povoamentos puros e sua influência sobre atributos edáficos em Trajano de Moraes, RJ. 2012. $64 \mathrm{f}$. Dissertação (Mestrado em Produção Vegetal), Universidade Estadual do Norte Fluminense Darcy Ribeiro, Campos dos Goytacazes, 2012.

SPATHELF, P.; FLEIG, F. D.; VACCARO, S.; ESBER, L.M. Análise dendroecológica de Ocotea pulchella Nest et Mart. ex Nees (Canela-lageana) na Serra Geral de Santa Maria, RS, Brasil. Ciência Florestal, v. 10, n. 1, p. 95-108, 2000.

TITUS, S. J.; MORTON, R. T. Forest Stand Growth Models. The Forestry Chronicle, v. 61, p. 19- 22, 1988.

WISCH, E. N.; MELLO, A. C. G. Análise do crescimento de cinco espécies nativas do cerrado no oeste do estado de São Paulo. Revista Científica Eletrônica de Engenharia Florestal, n.5, 2005. Disponível em: $<$ http://faef.revista.inf.br/site/e/engenharia-florestal-5-edicao-fevereiro-de2005.html\#tab171>. Acesso em: 24 de março de 2016.

ZANIN, N. T. Modelagem da produção mensal de indivíduos de Araucaria angustifolia e Pinus taeda com inclusão de variáveis meteorológicas. 2013. 97 f. Dissertação (Mestrado em Ciências Florestais), Universidade Federal do Paraná, Curitiba, 2013. 